\title{
The Eos SAR Mission
}

Charles Elachi, Daren Casey, Benjamin Holt,

Wu Yang Tsai, Mike Sander and JoBea Way

Jet Propulsion Laboratory

Pasadena, Califomia 91001

\section{Abstract}

The Eos SAR if a key component of the Eos mission. It is currently being launched on a free flyer in parallel with Eos-A to provide coincident measurements of the Earth's surface over a 15 year time span. This paper provides the latest information on the status of the Eos SAR mission, emphasizes the SAR's role in the overall Eos mission, and compares the Eos SAR under study to the earlier SAR on Eos-B.

\section{Status}

The EOS SAR had been carried as a facility instrument on Eos-B through the June, 1989, NonAdvocacy Review (NAR). Shortly after the NAR was held, NASA elected to take the SAR off the Eos-B platform. This was done to make the designs of the Eos$A$ and the Eos-B platforms the same in order to reduce the cost of the Eos mission. Because the SAR drove most of the mass, data rate, and power requirements on Eos$B$, the instrument was removed. NASA then requested JPL to study a dedicated SAR mission tailored to the SAR requirements

JPL recently conducted a study and defined an Eos SAR mission on a dedicated Delta II launch. The revised SAR has almost the full capability of the original EOS SAR. Data will be input to EosDIS and available to the science community.

\section{The Role of SAR in Eos}

Global Warming and the Carbon Cycle

Atmospheric $\mathrm{CO}_{2}$ flux from deforestation is a key unknown parameter in determining the contribution of the carbon cycle to global warming. Unknown parameters include the areal extent of deforestation (measurable with a single channel radar) and the biomass of existing forests (only measurable with multichannel radar). Biomass is essential to determine carbon input from cleared forests. In their first $10-20$ years, forests reach maximum leaf area index in order to intercept maximum sun for growth. This shields the lower canopy to optical sensors. Beyond 20 years, forest growth occurs in the trunks and branches. Only the long wavelengths of SAR can penetrate the upper canopy and sense forest biomass. The other major unknown in carbon cycle is the amount of carbon taken up in the ocean by phytoplankton: MODIS on Eos$A$ will measure this. Thus both SAR and MODIS are essential to determine the role of the carbon cycle in global warming.

\section{Hydrologic Cycle}

Understanding the global hydrologic cycle is essential as it plays a role in nearly every process on Earth. A key component is soil moisture. Historically it has been difficult to measure soil moisture with any remote sensing instruments as surface roughness and vegetation confuse the measurements. New advances which utilize multipolarization radar techniques allow the separation and measurement of the soil and vegetation moisture independently. The SAR is the enly Eos instrument with the potential capability of global soil moisture measurement.

\section{Polar Ice Mapping}

Through its fine-scale measurement of sea ice motion, ice type and concentration, and ice edge configuration, the EOS SAR (in conjunction with MODIS surface temperature and ocean color data) is a key instrument for determining ice dynamics, heat flux bottom water formation and ice margin biological products. All of these measurements are important for modeling of the global climate, energy balance, and ocean circulation, and for monitoring ocean/atmosphere/ cryosphere/biosphere interactions. In addition, knowledge of the nature of thin sea ice as measured by the Eos SAR along with HIMSS will help assess the magnitude of global warming. Because of the lighting conditions and cloud cover in the polar regions, the radar, with its global coverage capability, is essential to achieve this monitoring capability. Several key algorithms, including ice motion and ice classification, have already been implemented and are operational at the Alaska SAR Facility (ASF) in preparation for ERS-1.

\section{The New Eos SAR}

The scientific measurements stated above require a multiple frequency (at least three), full polarization imaging radar with the capability for global mapping every four to five days at moderate resolution $(250 \mathrm{~m})$ with nested local high resolution (30 m) capability. This cannot be met by any U. S. or international SAR presently under development or planned (SIR-C/X-SAR, ERS-1, JERS-1 or Radarsat). A number of science advisory groups appointed by NASA recommended a multiparameter $S A R$ to be a key facility on Eos. 
The capabilities of the new Eos SAR under study and the previous Eos SAR are compared in Table 1. The new Eos SAR, like its predecessor, will have three

frequencies : L-, C-, and X-bands. However, as a result of the new mass, power, and launch envelope constraints, quad polarization will be available only at L-band, and dual-polarization available at $C$ - and $X$ bands. The swath width for the global mapping mode will be reduced from $600 \mathrm{~km}$ to approximately $350-450$ $\mathrm{km}$. The other modes will not be affected. A global coverage capability may still be achieved over five days at an altitude of $620 \mathrm{~km}$ with the reduced swath and maximum incidence angle $\left(40^{\circ}\right)$. The new altitude will reduce the amount of simultaneous data collected with MODIS; however, well over half of the MODIS data will be collected within 60 minutes of the SAR passing over the same target.

In the coming year, the Eos SAR Facility

Instrument Team will be reviewing the SAR science requirements and will then make recommendations with regard to the SAR capabilities.

\section{Su m m a r y}

The JPL study has shown that a dedicated Eos SAR mission is a technically and fiscally sensible approach. The SAR is a key element of the Eos program and is essential to make the measurements needed for global change studies. It is critical, especially for carbon cycle studies, that the SAR data are acquired in conjunction with the MODIS and HIRIS data on Eos-A (launch in 1997). Therefore, it is essential that the Eos SAR be launched in parallel with Eos-A.

Acknowledgements This work was carried out at the Jet Propulsion Laboratory, California Institute of Technology under contract to the National Aeronautics and Space Administration.

Table 1

Eos-B's Eos SAR

$\begin{array}{ll}\text { Frequencies : } & \begin{array}{l}\text { L-band }(1.2 \mathrm{GHz}, 25 \mathrm{~cm}) \\ \text { C-band }(5 \mathrm{GHz}, 6 \mathrm{~cm}) \\ \text { X-band }(9 \mathrm{GHz}, 3 \mathrm{~cm})\end{array} \\ \text { Polarization: } & \begin{array}{l}\text { Quad-polarization L-band } \\ \text { Quad-polarization C-band } \\ \text { Dual-polarization X-band }\end{array}\end{array}$

Imaging modes:

Global mapping

Regional mapping

Local high res'n
$250 \mathrm{~m}$ res'n, $600 \mathrm{~km}$ swath 50-100 $\mathrm{m}$ res' $\mathrm{n}, 100-200 \mathrm{~km}$ swath

$20-30 \mathrm{~m}$ res' $\mathrm{n}, 30-50 \mathrm{~km}$ swath
New Eos SAR

(on SAR platform)

L-band $(1.2 \mathrm{GHz}, 25 \mathrm{~cm})$

C-band $(5 \mathrm{GHz}, 6 \mathrm{~cm})$

$X$-band $(9 \mathrm{GHz}, 3 \mathrm{~cm})$

Quad-polarization L-band Dual-polarization C-band Dual-polarization X-band

$250 \mathrm{~m}$ res' $\mathrm{n}, 350-450 \mathrm{~km}$ swath

$50-100 \mathrm{~m}$ res'n, $100-200 \mathrm{~km}$

swath

20-30 m res'n, $30-50 \mathrm{~km}$

swath 